\title{
The discovery of elements 113 to 118
}

\author{
Vladimir Utyonkov ${ }^{1, a}$, Yuri Oganessian ${ }^{1}$, Sergey Dmitriev ${ }^{1}$, Mikhail Itkis ${ }^{1}$, Kenton Moody ${ }^{2}$,

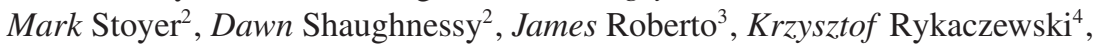 \\ and Joseph Hamilton ${ }^{5}$ for the collaboration H, $^{1,3,4,5,6,7}$ \\ ${ }^{1}$ Flerov Laboratory of Nuclear Reactions, Joint Institute for Nuclear Research, 141980 Dubna, \\ Russian Federation \\ ${ }^{2}$ Nuclear and Chemical Sciences Division, Lawrence Livermore National Laboratory, Livermore, \\ California 94551, USA \\ ${ }^{3}$ Science and Technology Partnerships Directorate, Oak Ridge National Laboratory, Oak Ridge, \\ Tennessee 37831, USA \\ ${ }^{4}$ Physics Division, Oak Ridge National Laboratory, Oak Ridge, Tennessee 37831, USA \\ ${ }^{5}$ Department of Physics and Astronomy, Vanderbilt University, Nashville, Tennessee 37235, USA \\ ${ }^{6}$ Department of Physics and Astronomy, University of Tennessee, Knoxville, Tennessee 37996, USA \\ ${ }^{7}$ Research Institute of Atomic Reactors, 433510 Dimitrovgrad, Russian Federation
}

\begin{abstract}
Discovery and investigation of the "Island of stability" of superheavy nuclei at the separator DGFRS in the ${ }^{238} \mathrm{U}-{ }^{249} \mathrm{Cf}+{ }^{48} \mathrm{Ca}$ reactions is reviewed. The results are compared with the data obtained in chemistry experiments and at the separators SHIP, BGS, TASCA, and GARIS. The synthesis of the heaviest nuclei, their decay properties, and methods of identification are discussed and compared with the criteria that must be satisfied for claiming the discovery of a new chemical element. The role of shell effects in the stability of superheavy nuclei is demonstrated by comparison of the experimental results with empirical systematics and theoretical data.
\end{abstract}

\section{Introduction}

For half a century, various theoretical models have predicted the existence of an area of enhanced stability of heaviest (superheavy) nuclei (SHN). A group of nuclei in the vicinity of the predicted doubly magic spherical nucleus ${ }^{298} 114_{184}$ were named superheavy. Just after the appearance of the first theoretical predictions of this area of SHN, numerous attempts were undertaken to synthesize them artificially; however, all the efforts were in vain. Later on, it became clear that in order to synthesize short-lived $(\leq 1 \mathrm{~min})$ SHN the sensitivity of experiments should be increased by two to three orders of magnitude.

In 1998, experiments aimed at the synthesis of SHN were initiated in FLNR, JINR. In these experiments, we employed the Dubna gas-filled recoil separator (DGRFS), which allows the separation of the products of complete fusion reactions from the beam of bombarding ions, elastically-scattered nuclei, and products of incomplete fusion; with the subsequent collection of the complete fusion products in a detection system. The detection system includes proportional chambers used to measure the time of flight (TOF) of particles and several semiconductor detectors; the latter are separated in position-sensitive strips.

\footnotetext{
${ }^{a}$ Corresponding author: utyonkov@jinr.ru
} 
Table 1. Production and radioactive properties [4] used for identification of nuclei.

\begin{tabular}{|c|c|}
\hline Production properties $^{a}$ & Radioactive properties $^{\mathrm{a}}$ \\
\hline Energy of bombarding particles, $\mathrm{C}$ & Kind of decay, C \\
\hline Cross section, $\mathrm{C}$ & Branching ratio, $\mathrm{C}$ \\
\hline Yield curve, $\mathrm{C}, \mathrm{A}(A, Z)$ & Half-life, C \\
\hline Cross bombardments, $\mathrm{C}, \mathrm{A}(A, Z)$ & Energy of $\alpha$-particles, $C$ \\
\hline Angular distribution ${ }^{\mathrm{b}}, \mathrm{A}(Z)$ & Maximum energy of $\beta$-particles ${ }^{\mathfrak{c}}, \mathrm{C}$ \\
\hline Angular selection, $\mathrm{A}(Z)$ & Energy of $\gamma$-radiations ${ }^{\mathrm{c}}, \mathrm{C}$ \\
\hline Mass separation ${ }^{\mathrm{d}}, \mathrm{A}(A)$ & $X$-ray spectrum $(K \text { or } L)^{\mathrm{c}}, \mathrm{C}, \mathrm{A}(Z)$ \\
\hline Velocity filter (separator), $\mathrm{A}(Z)$ & Fission characteristics, $\mathrm{C}$ \\
\hline Time of flight selection, $\mathrm{A}(Z)$ & $\begin{array}{l}\text { Genetic relation between ancestor and } \mathrm{n}^{\text {th }} \\
\text { generation descendant }{ }^{\mathrm{b}}, \mathrm{C}, \mathrm{A}(Z)\end{array}$ \\
\hline Chemistry, $\mathrm{A}(Z)$ & \\
\hline
\end{tabular}

\footnotetext{
${ }^{a} \mathrm{C}$ - characterization property, $\mathrm{A}$ - assignment property: $\mathrm{A}(Z)$ for $Z, \mathrm{~A}(A)$ for $A$, and $\mathrm{A}(A, Z)$ for both.

${ }^{\mathrm{b}}$ If combined with chemistry experiments.

${ }^{\mathrm{c}}$ Was not applied.

${ }^{\mathrm{d}}$ With limited mass resolution.
}

During 15 years of experiments with the DGFRS, six new superheavy elements located on the shore of the "Island of stability" and having atomic numbers 113 through 118 were synthesized for the first time. Radioactive properties of more than fifty new heaviest nuclides were explored in the experiments carried out in collaboration with the laboratories of the USA in Livermore (LLNL), Oak Ridge (ORNL), Knoxville (UT), Nashville (VU) and with Russian centers RIAR (Dimitrovgrad) and RIEPh (Sarov) [1-3].

Radioactive properties of the synthesized nuclides demonstrate a substantial increase of nuclear stability with increase of neutron number and closer approach to the predicted spherical shells $Z=114$ (or at even higher $Z$, such as 120,124 or 126) and $N=184$. New observations establish a consistent pattern of nuclear properties in the area of the heaviest nuclides. They demonstrate the decisive role of nuclear shells and provide experimental proof of the existence of the predicted "Island of stability" of superheavy elements.

\section{Experimental results}

Decay chains of all of the nuclei synthesized hitherto in the ${ }^{238} \mathrm{U}-{ }^{249} \mathrm{Cf}+{ }^{48} \mathrm{Ca}$ reactions are terminated by spontaneous fission (SF). Thus, the region of SHN is not yet linked to known isotopes; here, the method of the detection of consecutive $\alpha$ decays that lead to the known nuclei can be applied for the identification of the new ones only after independent identification of at least one isotope of a given element in the decay chain, e.g., by chemical identification of its atomic number. However, in addition to this method, approximately 20 other criteria were developed by IUPAC/IUPAP in 1991 "that must be satisfied for the discovery of a new chemical element to be recognized" [4]. The criteria were subdivided into Production properties and Radioactive properties: "The first establishes physical and/or chemical properties of samples suspected of containing the new element and that are sufficient to categorize it. The second extends to properties that are used to demonstrate that the "characterization properties" are indeed those of an unknown element. Some properties can be used for both purposes." Most of these criteria demonstrate, beyond a reasonable doubt, the synthesis of the nuclides with atomic numbers $Z=113-118$ (see Table 1).

Characteristics of the DGFRS that is used in the experiments meet the following requirements. The principle of operation of the separator is selection of products of the complete-fusion reaction by their charge state $(q)$ in a rare gas and kinematic characteristics 


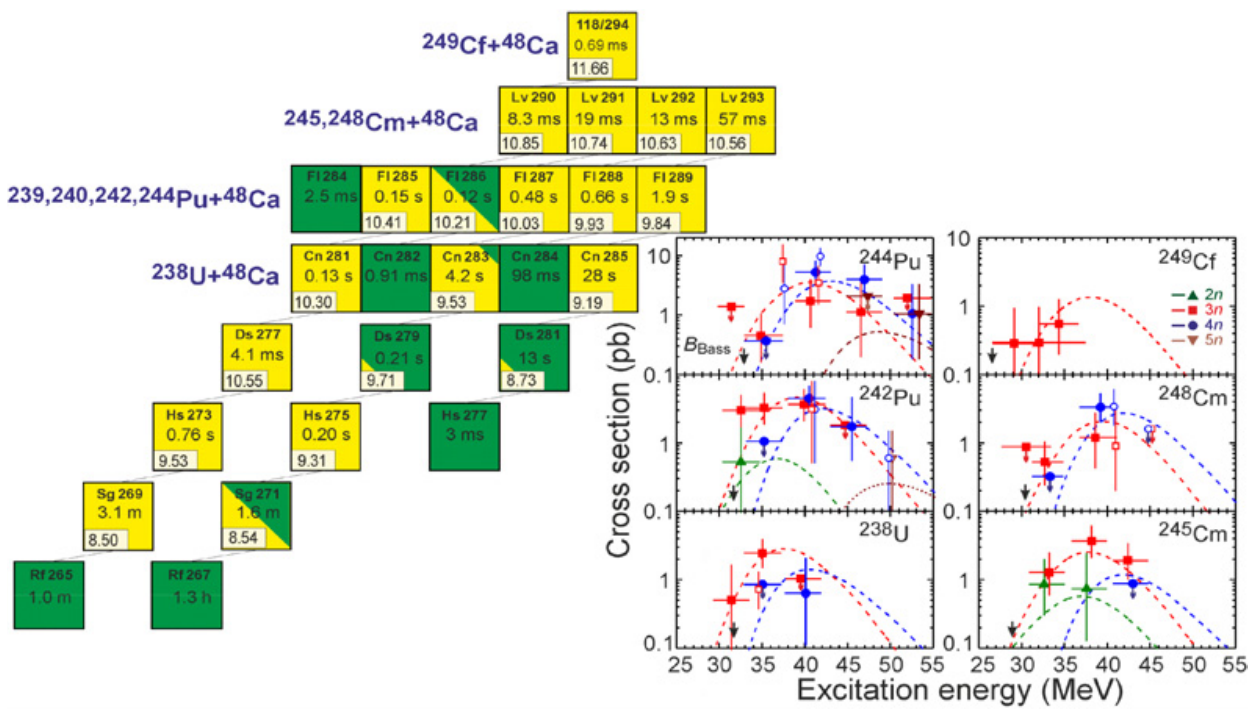

Figure 1. Left-hand side: summary of decay properties of isotopes of even- $Z$ elements synthesized in the given reactions. The average energies of $\alpha$ particles and half-lives are given for $\alpha$ emitters (yellow squares). For spontaneously fissioning nuclei marked by green squares the half-lives are listed. Righthand side: Excitation functions for the $2 n$ - to $5 n$-evaporation channels (see explanations in text) from the complete-fusion reactions ${ }^{238} \mathrm{U}-{ }^{249} \mathrm{Cf}+{ }^{48} \mathrm{Ca}$ measured at DGFRS (solid symbols) and SHIP, BGS, and TASCA (open symbols). Dotted lines show results of calculations [7]. Vertical error bars correspond to statistical uncertainties of the DGFRS experiments and available data from other experiments. Upper cross-section limits are shown by arrows. Horizontal error bars represent the range of excitation energies corresponding to a given beam energy. For reference purposes we show the energy at the Bass barrier $\left(B_{\text {Bass }}\right)[8]$ (black arrow).

(mass of recoil nucleus $(m)$ and its velocity $(v)$ ) in accordance with the separator magnetic rigidity $B \rho=m v / q$. Note that $q$ depends linearly on $v$. These values are calculated for the $x n$-reaction channel when setting the separator's parameters. The setup (separator) strongly separates forward-peaked evaporation residues (ER), products of complete-fusion reactions, within a narrow angle $\pm 2-3^{\circ}$ ("angular selection") with a huge suppression of the products of the transfer reactions and even incomplete fusion, e.g., $\alpha x n$ reactions ("mass separation" with limited resolution). The TOF selection in the existing separators may be substituted by the equivalent combined measurement of recoil energy and TOF. Note, the production properties discussed here were called "assignment properties" in [4].

The first superheavy nucleus ${ }^{289} \mathrm{Fl}$ was discovered in 1999 in the ${ }^{244} \mathrm{Pu}\left({ }^{48} \mathrm{Ca}, 3 n\right)$ reaction studied at DGFRS (here and after we refer to reviews [1-3] containing references to most of earlier experimental data). The decay properties of ${ }^{289} \mathrm{Fl}$ and descendant nuclei ${ }^{285} \mathrm{Cn},{ }^{281} \mathrm{Ds}$, and ${ }^{277} \mathrm{Hs}$, later observed at the TASCA separator [5], are shown in Fig. 1.

With an increase of the excitation energy $E^{*}$ of the compound nucleus $(\mathrm{CN})$, the yields of different nuclides vary ("at increasingly higher energy values for increasing values of $x$ " [4]). At the lowest $E^{*}$ values, the nuclides with relatively lower $\alpha$-particle energies and longer half-lives $T_{1 / 2}$ are produced (viz., ${ }^{289} \mathrm{Fl}$ ). At larger projectile energies, the yield of these nuclei decreases, but the yield of other nuclides $\left({ }^{288} \mathrm{Fl}\right.$ and $\left.{ }^{287} \mathrm{Fl}\right)$, with higher $E_{\alpha}$ and shorter $T_{1 / 2}$ values (by about $0.1 \mathrm{MeV}$ and by factor of about two, respectively, for parent nuclei), increases and then decreases again with the further increase of the excitation energy, see Fig. 1. The decay properties of the nuclides, consecutively appearing with the rise of $E^{*}$, are different (compare the $E_{\alpha}$ and $T_{1 / 2}$ values and decay modes of all of the nuclei in 
the chains in Fig. 1). In particular, the decay chains of even- $Z$, odd- $N$ nuclei are longer indicating the hindrance of fission process as compared to the even-even nuclei decays. However, the difference in $E_{\alpha}$ and $T_{1 / 2}$ values of parent nuclei ${ }^{287-289} \mathrm{Fl}$ is rather small; this observation excludes the assignment of the observed nuclides to differing types of reactions (e.g., considering that one isotope is produced in the $x n$ channel and others - in the pxn or axn channels).

The production cross-section values as well as energies of bombarding particles (see items in Table 1) are comparable for all of the studied reactions, which might indicate the identity of the mechanism of all of the reactions. In Fig. 1, the excitation energy values were calculated from mass tables [9]. One could use other mass tables which would result in a shift of all of the data points by a couple of $\mathrm{MeV}$. However, the excitation energy relative to the Bass barriers ( $a$ value independent of mass predictions) is firmly fixed.

For synthesis of the lighter isotopes of $\mathrm{Fl}$, the reactions with lighter target nuclei were studied at DGFRS [1-3]. In the ${ }^{242} \mathrm{Pu}+{ }^{48} \mathrm{Ca}$ reaction, the isotope ${ }^{287} \mathrm{Fl}$ was predominantly observed at low excitation energies while the lighter isotope $\left({ }^{286} \mathrm{Fl}\right)$ was obtained at higher projectile energies. Further increase of ${ }^{48} \mathrm{Ca}$ energy resulted in observation of the next isotope ${ }^{285} \mathrm{Fl}$, first at BGS [10]. The same isotope was also produced at DGFRS in the reaction with a lighter $\mathrm{Pu}$ isotope as the product of the ${ }^{240} \mathrm{Pu}\left({ }^{48} \mathrm{Ca}, 3 n\right){ }^{285} \mathrm{Fl}$ reaction [11]. In the same experiment carried out at higher ${ }^{48} \mathrm{Ca}$ energy as well as in the ${ }^{239} \mathrm{Pu}+{ }^{48} \mathrm{Ca}$ reaction, a spontaneously fissioning nuclide was observed and assigned to the new isotope ${ }^{284} \mathrm{Fl}$.

The excitation functions (item "yield curve" in Table 1) have been measured for the reactions with ${ }^{244} \mathrm{Pu}$ and ${ }^{242} \mathrm{Pu}$ in the $E^{*}$ interval of about $32-53 \mathrm{MeV}$ and at $E^{*}=39$ and $43 \mathrm{MeV}$ in the reaction with ${ }^{240} \mathrm{Pu}$ (Fig. 1), which, together with decay properties of the observed nuclides (items "kind of decay", "branching ratio", "half-life", and " $\alpha$-particle energy" in Table 1), demonstrate the production of the neighbouring isotopes of the same element in each reaction.

Two $\mathrm{Fl}$ isotopes as well as their descendant nuclei were observed in cross bombardments ${ }^{244} \mathrm{Pu}\left({ }^{48} \mathrm{Ca}, 5 n\right){ }^{287} \mathrm{Fl}, \quad{ }^{242} \mathrm{Pu}\left({ }^{48} \mathrm{Ca}, 3 n\right){ }^{287} \mathrm{Fl},{ }^{242} \mathrm{Pu}\left({ }^{48} \mathrm{Ca}, 5 n\right){ }^{285} \mathrm{Fl}$, and ${ }^{240} \mathrm{Pu}\left({ }^{48} \mathrm{Ca}, 3 n\right){ }^{285} \mathrm{Fl}$. Moreover, Cn isotopes ${ }^{282} \mathrm{Cn}$ and ${ }^{283} \mathrm{Cn}$, daughter nuclei of ${ }^{286} \mathrm{Fl}$ and ${ }^{287} \mathrm{Fl}$, were produced in another cross-bombardment reaction ${ }^{238} \mathrm{U}+{ }^{48} \mathrm{Ca}$. This proves that these nuclei were produced in the same type of reaction - xn, pxn, $\alpha x n$, and so on. Indeed, with increase of the atomic (by two protons, e.g., from ${ }^{238} \mathrm{U}$ to ${ }^{242} \mathrm{Pu}$ or ${ }^{244} \mathrm{Pu}$ ) and mass numbers of the target nuclei, heavier parent nuclides are produced; their $\alpha$ decay leads to nuclei that were synthesized in reactions with lower- $Z$ and lighter target nuclei. Another example of the cross bombardments is the observation of the same nuclides in reactions with target isotopes of the same $Z$ but with different mass numbers. If the target isotopes differ by several neutrons, e.g., ${ }^{242} \mathrm{Pu}$ and ${ }^{244} \mathrm{Pu}$ (or ${ }^{240} \mathrm{Pu}$ and ${ }^{242} \mathrm{Pu}$ ), then the observed parent nuclide, e.g. ${ }^{287} \mathrm{Fl}$ ( or ${ }^{285} \mathrm{Fl}$ ), can be produced in the $3 n$-evaporation channel of the reaction, with ${ }^{242} \mathrm{Pu}\left(\right.$ or $\left.{ }^{240} \mathrm{Pu}\right)$ at a lower excitation energy, and in the $5 n$ channel of the reaction, with ${ }^{244} \mathrm{Pu}\left(\right.$ or $\left.{ }^{242} \mathrm{Pu}\right)$ at higher excitation energy. Here, the identity of the decay properties observed in cross bombardments definitely proves that the same nucleus was produced in two reaction channels that differ merely by the number of evaporated neutrons.

The first nucleus of element $116,{ }^{293} \mathrm{Lv}$, was synthesized at DGFRS in 2000 in the ${ }^{248} \mathrm{Cm}+{ }^{48} \mathrm{Ca}$ reaction [1-3]. This isotope was observed on the low-energy side of the excitation function. Increasing the excitation energy of the $\mathrm{CN}$ resulted in the observation of two isotopes: one already observed at lower energy ${ }^{293} \mathrm{Lv}$ and a new lighter isotope ${ }^{292} \mathrm{Lv}$. Two even lighter isotopes, ${ }^{291} \mathrm{Lv}$ and ${ }^{290} \mathrm{Lv}$, were produced in the reaction of ${ }^{48} \mathrm{Ca}$ with the lighter target isotope ${ }^{245} \mathrm{Cm}$ followed by evaporation of two and three neutrons: ${ }^{245} \mathrm{Cm}\left({ }^{48} \mathrm{Ca}, 2 n\right){ }^{291} \mathrm{Lv}$ and ${ }^{245} \mathrm{Cm}\left({ }^{48} \mathrm{Ca}, 3 n\right){ }^{290} \mathrm{Lv}$. The decay properties of descendants of the 

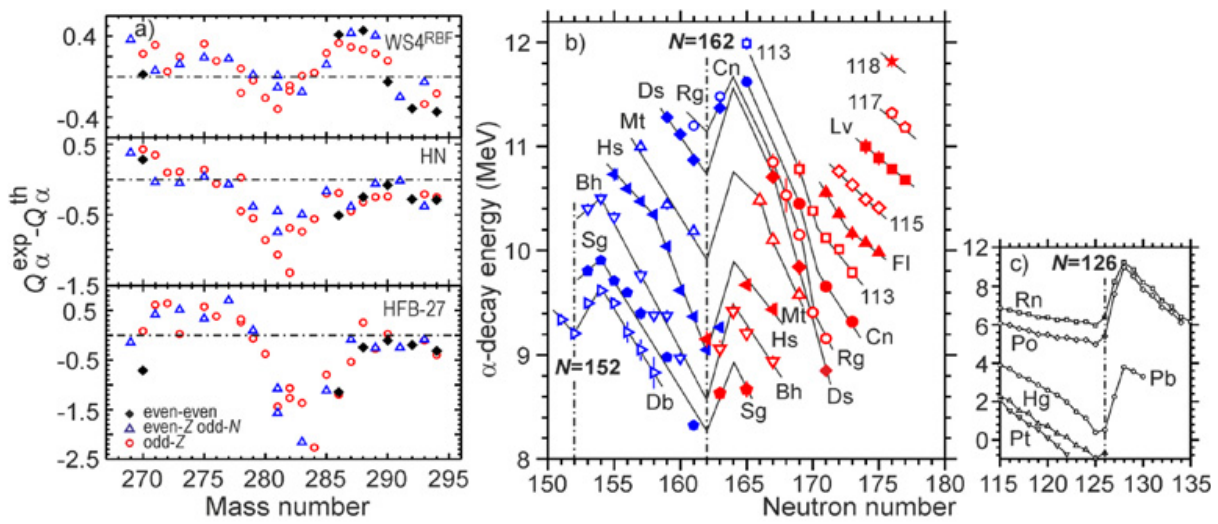

Figure 2. (a) Deviation between experimental and calculated (see Fig.12 in [3]) $\alpha$-decay energies for even-even, even- $Z$ and odd- $N$, and odd- $Z$ nuclei. (b) Measured $\alpha$-decay energy vs. neutron number for the isotopes of elements 106-118 (filled and open symbols refer to even- $Z$ and odd- $Z$ nuclei, respectively; $Q_{\alpha}$ values for nuclei produced in the Ra-Cf $+{ }^{48} \mathrm{Ca}$ reactions are shown in red; other data (blue symbols) are taken from [12]. The lines are drawn to guide the eye. (c) The $Q_{\alpha}$ values for isotopes of even- $Z$ elements Pt-Rn [12] are shown for comparison.

isotopes ${ }^{290} \mathrm{Lv}-{ }^{293} \mathrm{Lv}$ were in full agreement with those found in the ${ }^{244} \mathrm{Pu}+{ }^{48} \mathrm{Ca},{ }^{242} \mathrm{Pu}+{ }^{48} \mathrm{Ca}$, and ${ }^{238} \mathrm{U}+{ }^{48} \mathrm{Ca}$ cross-bombardment reactions.

The first decay chain of element 118 was found in 2002 in the ${ }^{249} \mathrm{Cf}\left({ }^{48} \mathrm{Ca}, 3 n\right){ }^{294} 118$ reaction studied at DGFRS [1-3]. Here one should note that the descendant SF isotope ${ }^{282} \mathrm{Cn}$ was produced in the following cross reactions: ${ }^{238} \mathrm{U}\left({ }^{48} \mathrm{Ca}, 4 n\right){ }^{282} \mathrm{Cn},{ }^{242} \mathrm{Pu}\left({ }^{48} \mathrm{Ca}, 4 n\right){ }^{286} \mathrm{Fl}-(\alpha)$ ${ }^{282} \mathrm{Cn},{ }^{245} \mathrm{Cm}\left({ }^{48} \mathrm{Ca}, 3 n\right){ }^{290} \mathrm{Lv}-(\alpha, \alpha)-{ }^{282} \mathrm{Cn}$, and ${ }^{249} \mathrm{Cf}\left({ }^{48} \mathrm{Ca}, 3 n\right){ }^{294} 118-(\alpha, \alpha, \alpha)-{ }^{282} \mathrm{Cn}$.

Assignment of all of the synthesized nuclides to the products of the $x n$-reaction channel is justified by characteristics of the DGFRS, excitation-function measurements, production of most of the nuclides in cross bombardments, as well as by decay properties of nuclei, especially their measured $\alpha$-particle energies [see Fig. 2(b)].

The population of the relatively high-energy levels in the parent nucleus followed by $\alpha$ decay to the low-energy levels or the ground state of the daughter nucleus leads to a higher $\alpha$-particle energy $E_{\alpha}$ than that corresponding to the ground-state-to-ground-state $\alpha$-decay energy $Q_{\alpha}$. However, the following $\alpha$ decay starts from a low-energy level. Thus, the observation of decays from high- to low-energy levels for all of the nuclei in the long decay chains (e.g., ${ }^{285} \mathrm{Fl},{ }^{291} \mathrm{Lv}$, or odd- $Z$ nuclei, see below) is absolutely excluded. Vice versa, $\alpha$ decay can go to excited states of the daughter nucleus, followed by the emission of $\gamma$ rays, which results in a lower $E_{\alpha}$ than that corresponding to the $Q_{\alpha}$ value. The even- $Z$ isotopes investigated so far do not indicate $\alpha$ decays from the excited (isomeric) states (see Fig. 1 and $[2,3])$; their $\alpha$-particle energies are identical and form single peaks in both cases - after production of nuclei in primary reactions and after $\alpha$ decays of parent nuclei (with several lower-energy peaks observed for even-odd ${ }^{291} \mathrm{Lv},{ }^{289} \mathrm{Fl}$, and ${ }^{283} \mathrm{Cn}$ ). Calculation of the $Q_{\alpha}$ values from $\alpha$ peaks appears to be reasonable for even-even isotopes as well as for even- $Z$ odd- $N$ nuclei because the energies of odd isotopes have intermediate values between those of even-even nuclides, which is in agreement with the observations for the lighter nuclei (see Figs. 21-26 in [12]).

An important feature observed in these experiments (see Fig. 2) is the fact that $Q_{\alpha}(N)$ systematics for isotopes of neighbouring elements never intersect (see also Figs. 25-26 in [12]). Moreover, the $Q_{\alpha}$ values for isotopes of $\mathrm{Sg}$ and $\mathrm{Hs}$ perfectly follow the trend 


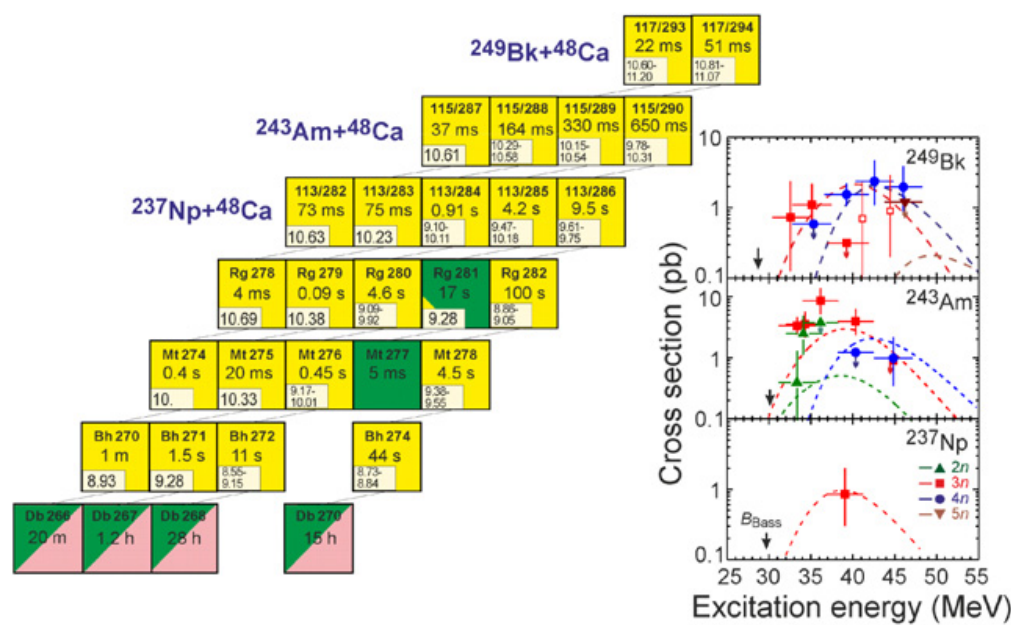

Figure 3. Same as Fig. 1, but for the isotopes of odd- $Z$ elements. For Db isotopes only SF was observed however, electron capture (EC) cannot be excluded.

of variation of $Q_{\alpha}(N)$ at the crossing of the magic neutron number $N=162$ (identical to what is observed for the isotopes of Ds and $\mathrm{Rg}$ as well as for numerous nuclides with $N \approx 152$ and 126, see Fig. 2c. Therefore, the systematics of $\alpha$-decay energies of even- $Z$ elements and their descendants provides proof of identification of the $Z$ and $N$ of these elements. In addition, the mass numbers of nuclei can be easily established by considering their decay properties (e.g., SF is much more probable for even- $N$ and/or $Z$ isotopes, see Figs. 1 and 3 below) and excitation functions (e.g., products of the ${ }^{244} \mathrm{Pu}+{ }^{48} \mathrm{Ca}$ might be assigned to the $1 n$ - to $3 n$-evaporation channel instead of $3 n$ - to $5 n$, but in this assumption, the product of the ${ }^{245} \mathrm{Cm}\left({ }^{48} \mathrm{Ca}, 2 n\right){ }^{291} \mathrm{Lv}$ reaction should originate from the radiative-capture channel, which was not registered even in the cold-fusion reactions of heavy ions with ${ }^{208} \mathrm{~Pb}$ and ${ }^{209} \mathrm{Bi}$ ).

Furthermore, the chemical characterization of $\mathrm{Cn}$, based on the direct comparison of the adsorption characteristics of atoms of ${ }^{283} \mathrm{Cn}$ to that of $\mathrm{Hg}$ and $\mathrm{Rn}$, was obtained. The results of these experiments "establish element 112 as a typical element of group 12" [15]. Thus, the atomic numbers of all of the isotopes in the decay chain, starting with ${ }^{291} \mathrm{Lv}$ and ending with ${ }^{267} \mathrm{Rf}$, were determined by the genetic relation (Table 1 ).

In all of the reactions with even- $Z$ target nuclei, two to three different parent nuclides were produced in the $2 n$ - to $5 n$-evaporation channels, except for the ${ }^{249} \mathrm{Cf}+{ }^{48} \mathrm{Ca}$ reaction which was studied at $E^{*}$ values lower than those corresponding to the predicted cross-section maximum. Thus, it would be reasonable to expect similar observations for the reactions with odd- $Z$ target nuclei. With the aim of synthesis of isotopes of elements 113,115 and 117 three reactions using odd $Z$ actinide target materials were investigated: ${ }^{237} \mathrm{~Np},{ }^{243} \mathrm{Am}$, ${ }^{249} \mathrm{Bk}+{ }^{48} \mathrm{Ca}[1-3]$ (see Fig. 3).

The heaviest odd $-Z$ nucleus was synthesized in the ${ }^{249} \mathrm{Bk}\left({ }^{48} \mathrm{Ca}, 3 n\right){ }^{294} 117$ reaction at low projectile energies in 2009 [2, 3]. At higher excitation energy we registered shorter decay chains of the even- $N$ isotope ${ }^{293} 117$; each consisted of three consecutive $\alpha$ decays terminated by $\mathrm{SF}$ of ${ }^{281} \mathrm{Rg}$ or $5-\mathrm{ms}{ }^{277} \mathrm{Mt}$ instead of $\alpha$ decay observed for their odd- $N$ neighbours ${ }^{280,282} \mathrm{Rg}$ and ${ }^{276,278} \mathrm{Mt}$. The nuclei ${ }^{277} \mathrm{Mt}(N=168)$ and ${ }^{281} \mathrm{Rg}(N=170)$ belong to the region with the lowest stability with respect to SF observed for even- $Z$ nuclei (Fig. 1). Accordingly, even the high hindrance gouverned by the characteristic of odd particles does 
not "save" these odd nuclei $\left({ }^{281} \mathrm{Rg},{ }^{277} \mathrm{Mt}\right)$ from SF, which is caused by the weakening of the stabilizing effect of the neutron shells at $N=162$ and $N=184$ (see Refs. in [1-3]).

The observations of elements 113 and 115 were first reported in 2003-2004 [1-3]. In the reaction ${ }^{243} \mathrm{Am}+{ }^{48} \mathrm{Ca}$, these two new elements were simultaneously synthesized for the first time. In 2010-2012, new series of experiments with ${ }^{243}$ Am were performed at DGFRS aimed at the measurement of the excitation function in a wider energy range. At the lowest ${ }^{48} \mathrm{Ca}$ energies, we detected decay chains consisting of two consecutive $\alpha$ decays ending in SF. Similar $\alpha-\alpha$-SF decay chains were observed for descendant nuclei in the chain starting with the isotope ${ }^{293} 117$. Therefore, assignment of these chains to the product of the $2 n$-reaction channel, ${ }^{289} 115$, seems to be the most reasonable. Note, the product of the $2 n$-reaction channel was evidently observed with the same yield in the ${ }^{245} \mathrm{Cm}\left({ }^{48} \mathrm{Ca}, 2 n\right){ }^{291} \mathrm{Lv}$ reaction [1-3]. At higher projectile energies, decay chains of ${ }^{288} 115$, the product of evaporation of three neutrons, were registered that undergo five $\alpha$ decays followed by SF. At the highest bombarding energy, we detected decay chains of ${ }^{287} 115$.

The same decay chains were later produced in experiments with ${ }^{243} \mathrm{Am}$ which were performed at TASCA [16] and BGS [17]. In [18], the summary of decay times of nuclei in the short decay chains were re-analyzed which led to the interpretation that some of these chains might start from the isotope ${ }^{288} 115$ and proceed through either SF or electron capture decay branches of ${ }^{284} 113$ and ${ }^{280} \mathrm{Rg}$. However, authors concluded that "clearly, more ... data ... are needed to verify any proposed decay scenario of ${ }^{288,289} 115^{\prime \prime}$.

For investigation of the region of neutron-deficient odd- $Z$ SHN, we studied the ${ }^{237} \mathrm{~Np}\left({ }^{48} \mathrm{Ca}, 3 n\right){ }^{282} 113$ reaction. The decay properties of this isotope and its descendant nuclei ${ }^{278} \mathrm{Rg},{ }^{274} \mathrm{Mt},{ }^{270} \mathrm{Bh}$ and ${ }^{266} \mathrm{Db}$ are in agreement with those expected from the extrapolation of the radioactive decay properties of the heavier neighbouring isotopes ${ }^{283,284} 113$ and, respectively, their descendants.

Similar to even- $Z$ elements, the assignment of all of the odd- $Z$ nuclides to the products of the $x n$-reaction channel is based on characteristics of the DGFRS, excitation-function measurements for the reactions with ${ }^{243} \mathrm{Am}$ and ${ }^{249} \mathrm{Bk}$ ( $2 n$ - to $4 n$-evaporation channels were observed), and production of ${ }^{289} 115$ in cross bombardments, as well as decay properties of nuclei. First of all, the decay properties of all of the odd- $Z$ nuclei evidently differ from those observed for ${ }^{290-293} \mathrm{Lv},{ }^{285-289} \mathrm{Fl}$, etc. (potential products of the pxn-reaction channels or EC of parent nuclei).

For odd- $Z$ nuclei, the $\alpha$ spectra are more complex, which makes estimations of $Q_{\alpha}$ more difficult. However, the calculation of their $\alpha$-decay energies from the highest measured $\alpha$-particle energies [1-3] appears to be justified. For example, the $Q_{\alpha}$ values for odd-odd nuclei ${ }^{288} 115-{ }^{272} \mathrm{Bh}$, estimated or measured from the observed $\alpha-\gamma$ coincidences $[16,17]$ are in good agreement with such calculations. For all of the nuclides, these values are lower than those from $[16,17]$ by $0.03-0.24 \mathrm{MeV}$ [3], which might indicate that transitions occur to the low-lying exited states of the descendants. Thus, the calculated $Q_{\alpha}$ values for odd- $Z$ nuclei (Fig. 2) can represent their lower limits, which can differ from the exact $Q_{\alpha}$ values by a few hundred keV at most. The systematics shown in Fig. $2 b$ demonstrates that the $\alpha$-decay energies of all of the odd- $Z$ nuclei are located in between and are in good agreement with the interpolations/extrapolations from neighbouring even- $Z$ nuclides with $(Z \pm 1)$ and the same neutron number. The $Q_{\alpha}$ values for Bh isotopes are located between $Q_{\alpha}(N)$ systematics for $\mathrm{Sg}$ and $\mathrm{Hs}$ and perfectly correspond to the pattern of variation of $Q_{\alpha}(N)$ for Bh isotopes at the crossing of $N=162$.

Moreover, the terminal SF nuclide in the decay chain of ${ }^{288} 115$ produced in the ${ }^{243} \mathrm{Am}+{ }^{48} \mathrm{Ca}$ reaction was chemically characterized as a transactinide element [1-3]. In these experiments, the products from the irradiation of ${ }^{243} \mathrm{Am}$ by ${ }^{48} \mathrm{Ca}$ projectiles, having the same 
${ }^{48} \mathrm{Ca}$ energy as in the DGFRS experiments, were collected within a larger acceptance angle of $\pm 12.5^{\circ}$ that would result in increase of collection efficiency for transfer-reaction products by a factor of more than twenty. Nevertheless, after chemical extraction of transactinide elements, the SF nuclide was observed with the same decay properties - decay mode, half-life, and total kinetic energy (TKE) - and with the same cross section as at the DGFRS. All of these factors allowed for the conclusion that the same isotope was observed in both the physical and chemical experiments [1-3]. Simultaneously, all of the precursors $(Z=107,109,111$, 113 , and 115) discovered in [1-3] were identified by establishing the genetic link between the ancestor and the descendant.

In addition one could mention that the properties of $\alpha$-decaying even-even SHN are in agreement with the empirical Geiger-Nuttall relationship (see Fig. 26 in [1]). For SF nuclei it is observed that, with the transition $Z \geq 110$, the TKE increases with increasing $Z$ in agreement with the previously established dependence of TKE vs. parameter $Z^{2} / A^{1 / 3}$, typical for the asymmetric fission of lighter nuclei (see Fig. 29 in [1]).

Therefore, conformity with all of the above-mentioned criteria demonstrates that the observed SHN originate from the complete-fusion reactions followed by the evaporation of the neutrons. Furthermore, the decay properties of ${ }^{294} 117,{ }^{291-293} \mathrm{Lv},{ }^{287-289} 115,{ }^{285-289} \mathrm{Fl}$, and ${ }^{283} \mathrm{Cn}$ and their descendants were determined in different laboratories with use of different techniques (separators DGFRS, SHIP, TASCA, BGS, GARIS, and chemistry setups) that is in accordance with demand of reproducibility for recognition of the discovery of new elements (see $[1,3,5,10,11,15-18]$, references therein, and proceedings of this Symposium).

\section{Discovery of superheavy nuclei}

As a result of the experiments performed with use of a beam of ${ }^{48} \mathrm{Ca}$ ions, the heaviest elements with atomic numbers 113-118 were synthesized. These elements fill the seventh period of the table by D.I. Mendeleev. In these investigations, more than fifty new nuclides, isotopes of elements 104-118 having the largest number of neutrons, were produced for the first time. Their decay properties were determined. The chart of the nuclei was essentially extended up to the nuclides with $Z=118\left({ }^{294} 118\right)$ and $N=177\left({ }^{294} 117\right.$ and $\left.{ }^{293} \mathrm{Lv}\right)$.

These nuclei demonstrate amazing vitality. The increase of the neutron number in nuclei with $N \geq 165$ results in a decrease of the $Q_{\alpha}$ energy (Fig. 2) and a considerable increase of their half-lives (Fig. 4). An especially strong growth of $T_{\alpha}(N)$ with increasing $N$ is observed for the isotopes of elements 109-113.

The maxima of the total cross sections, $\sigma_{x n}$, for the fusion-evaporation ${ }^{48} \mathrm{Ca}$-induced reactions reach about $10 \mathrm{pb}$ for $Z=114$ and 115 nuclei, exceeding the values from extrapolation of $\sigma_{x n}$ systematics for hot-fusion reactions by about three orders of magnitude. Such relatively high cross sections are caused by the large survivability of the CN, which is directly related to the high fission barriers in SHN that appear due to nuclear shell effects.

Even-even isotopes of $\mathrm{Cn}$ and $\mathrm{Fl}$ with $N=170$ and 172 are located in a region where a steep rise of $T_{S F}(N)$ is predicted. The difference of two neutrons in these isotopes increases the SF partial half-life by two orders of magnitude. Similarly, the addition of two neutrons to the nucleus ${ }^{286} \mathrm{Fl}$ leads to an increase of the stability of ${ }^{288} \mathrm{Fl}$ with respect to SF by at least a factor of fifteen. For heavier even-even nuclei, ${ }^{288} \mathrm{Fl},{ }^{290} \mathrm{Lv}$, ${ }^{292} \mathrm{Lv}$, and ${ }^{294} 118$, SF was not detected due to the more considerable rise of stability with regard to SF compared to $\alpha$ decay, with the neutron number approaching the magic number $N=184$. On the other hand, the considerable drop of the evaporation cross sections observed for the reactions with neutron-deficient isotopes ${ }^{239} \mathrm{Pu}$ and ${ }^{240} \mathrm{Pu}$ (by a factors of about fifty and four, respectively, 


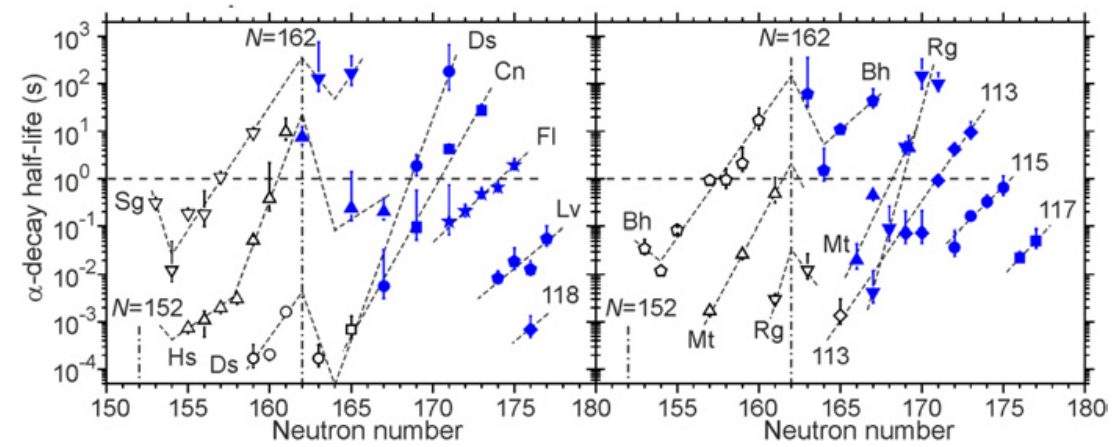

Figure 4. Half-lives vs. neutron number for the isotopes of even- $Z$ (left panel) and odd- $Z$ (right panel) elements with $Z=106-118$ [3] (results from $\mathrm{Ra}-\mathrm{Cf}+{ }^{48} \mathrm{Ca}$ reactions are shown by full blue symbols; other data are taken from [12]). Lines are drawn to guide the eye.

in comparison with that for the reaction with the heavier isotope ${ }^{244} \mathrm{Pu}$ [11]) indicates one is approaching the neutron-deficient border of stability of SHN.

In spite of the disagreement between different theoretical models in predicting the proton magic number which varies from 114 to 126 for SHN, the resulting calculated $\alpha$-decay energies, $Q_{\alpha}$, which are determined by nuclear masses, do not strongly deviate from experimental values. In Fig. 2a, the deviation between experiment and theory, $\Delta Q_{\alpha}=Q_{\alpha}^{\text {exp }}$ $Q_{\alpha}^{\text {th }}$, for even-even nuclei in all of the three cases is within approximately $\pm 0.5 \mathrm{MeV}$; however, the HFB-27 data systematically exceed the experimental $Q_{\alpha}$ values by approximately $0.5 \mathrm{MeV}$. The production cross-section values, calculated within different models (see Figs. 1, 2 and references given in [1-3]), as well as SF lifetimes of SHN (see, e.g., Fig. 5 in [11]) which are largely dependent on fission barriers of nuclei, are in reasonable agreement with experimental data. Therefore, the fundamental outcomes of the microscopic theory concerning the predictions of the "Island of stability" of SHN were validated by experimental evidence.

We acknowledge the support by the Russian Foundation for Basic Research, including recent Grants Nos. 13-02-12052, 13-03-12205, and 16-52-55002. Research at ORNL was supported by the U.S. DOE Office of Nuclear Physics under DOE Contract No. DE-AC05-00OR22725 with UT-Battelle, LLC. Research at LLNL was supported by LDRD Program Project No. 08-ERD-030, under DOE Contract No. DE-AC52-07NA27344 with Lawrence Livermore National Security, LLC. This work was also supported by the U.S. Department of Energy through Grant No. DE-FG-05-88ER40407 (Vanderbilt University). Most of the actinide target materials used in the DGFRS experiments were made at ORNL and provided through the DOE Isotope Program and at RIAR.

\section{References}

[1] Yu.Ts. Oganessian, J. Phys. G: Nucl. Part. Phys. 34, R165 (2007).

[2] Yu.Ts. Oganessian and V.K. Utyonkov, Rep. Prog. Phys. 78, 036301 (2015).

[3] Yu.Ts. Oganessian and V.K. Utyonkov, Nucl. Phys. A944, 62 (2015).

[4] A.H. Wapstra, Pure Appl. Chem. 63, 879 (1991).

[5] Ch.E. Düllmann et al., Phys. Rev. Lett. 104, 252701 (2010)

[6] J.M. Gates et al., Phys. Rev. C 83, 054618 (2011).

[7] V.I. Zagrebaev and W. Greiner, Nucl. Phys. A944, 257 (2015). 
[8] R. Bass, in: W. von Oertzen (ed.), Proc. Symp. Deep Inelastic and Fusion Reactions with Heavy Ions, in: Lecture Notes in Physics, vol. 117 (Springer-Verlag, Berlin, 1980) p. 281.

[9] W.D. Myers and W.J. Swiatecki, Nucl. Phys. A601, 141 (1996).

[10] P.A. Ellison et al., Phys. Rev. Lett. 105, 182701 (2010).

[11] V.K. Utyonkov et al., Phys. Rev. C 92, 034609 (2015).

[12] M. Wang et al., Chin. Phys. 36, 1603 (2012).

[13] G. Audi et al., Chin. Phys. 361287 (2012).

[14] G. Audi et al., Chin. Phys. 361157 (2012).

[15] R. Eichler et al., Nature 447, 72 (2007).

[16] D. Rudolph et al., Phys. Rev. Lett. 111, 112502 (2013).

[17] J.M. Gates et al., Phys. Rev. C 92, 021301(R) (2015).

[18] U. Forsberg et al., Nucl. Phys. A953, 117 (2016). 